\title{
Data Warehouse Implementation of Examination Databases
}

\author{
Muheet Ahmed Butt \\ Scientist Directorate of \\ Information Technology \& \\ Support Systems, University of \\ Kashmir, Srinagar, J\&K, India
}

\author{
S. M. K. Quadri \\ Head \& Director, \\ PG Department of Computer \\ Science, University of Kashmir, \\ Srinagar, Srinagar, J\&K, India
}

\author{
Majid Zaman \\ Scientist Directorate of \\ Information Technology \& \\ Support Systems, University of \\ Kashmir, Srinagar, J\&K, India
}

\begin{abstract}
A data warehouse is an asset for an enterprise and exists for the benefit of an entire enterpriseincluding business unit, individual customer, Student etc.Data in a data warehouse does not conform specifically to the preferences of any single enterprise entity. Instead, it is intended to provide data to the entire enterprise in such a way that all members can use the data in the warehouse throughout its lifespan [7]. This work explores using the star schema forAutomation of a Data Warehouse. An implementation of a data warehouse for an Examination Automation System is presented as an example.
\end{abstract}

\section{General Terms}

Data Warehouse, Star Schema, Examination Databases, Third Normal Form, Normalization, Dimension, Snowflake, Joins, Decision Support.

\section{Keywords}

Data Warehousing, Data Mining, Third Normal Form, Data Set.

\section{INTRODUCTION}

A 'data warehouse' is a repository of an organization's electronically stored data. Data warehouses are designed to facilitate reporting and analysis [5].This classic definition of the data warehouse focuses on data storage. However, the means to retrieve and analyze data, to extract, transform and load data, and to manage dictionary data are also considered essential components of a data warehousing system [11]. These operations depend more on the way the data is stored.

There are two leading approaches to storing data in a data warehouse

i. Dimensional approach and

ii. Normalized approach

In the dimensional approach, transaction data are partitioned into "facts", which are generally numeric transaction data, and"dimensions", which are the reference information that gives context to the facts [9]. A key advantage of a dimensional approach is that the data warehouse is easier for the user to understand and to use. The retrieval of data from the data warehouse also tends to operate very quickly. The main disadvantages of the dimensional approach are:

i. in order to maintain the integrity of facts and dimensions, loading of data from different operational systems is complicated, and ii. it is difficult to modify the data warehouse structure if the organization adopting the dimensional approach changes the way in which it does business.

In the normalized approach, the data in the data warehouse are stored following, to a degree, the Codd normalization rule. Tables are grouped together by subject areas that reflect general data categories. The main advantage of this approach is that it is very easy to add information into the database. A disadvantage of this approach is that because of the number of tables involved, it can be difficult for both users to join data from different sources into meaningful information and then access the information without a precise understanding of the sources of data and of the data structure of the data warehouse.

These approaches are not exact opposites of each other. Dimensional approaches can involve normalizing data to a degree [12].In this paper we have implemented a Star Schema Model of a Data Warehouse of an Central Automation of Examination System catering many colleges, Departments, Courses, Subjects, Subject Groups, Marks and tried to prepare results notifications at various levels which will enable us to build a build a Decision Support Database for future analysis.

The rest of the paper is organized as follows: Section 2 provides the information pertaining to various Data Warehouse Schemas used with their advantages. Section 3 provides the design of an example Data Warehouse for Examination Automation System giving detailed attribute information pertaining to the fact table. Section 4 provides the overall association of various dimensional table with the fact table. Section 5 provides the association of the fact Dimension of the Star Schema implementation for this example with other Dimensions in the schema.It also provides the results of the simulations of said implementation. Section 6 provides the means for aggregation of data present in the Star Schema Data Warehouse Design for Decision Support Systems. Section 7 provides brief description about the On-line Analytical Processing (OLAP) capabilities provided by the data warehouse or data mart. Section 8 provides the brief comparison between the $3^{\text {rd }}$ normal form and star schema implementation on the same test data. Conclusions drawn are depicted in Section 9. Section 10 lists the references and Appendix 1 provides the pictorial representation of the star schema and its relationship of fact tables with other dimensions. 


\section{SCHEMAS IN DATA WAREHOUSE}

A schema is a collection of database objects, including tables, views, indexes, and synonyms. There is a variety of ways of arranging schema objects in the schema models designed for data warehousing. The main database Schemas are:

\subsection{Star Schemas}

The star schema is perhaps the simplest data warehouse schema. It is called a star schema because the entityrelationship diagram of this schema resembles a star, with points radiating from a central table [6]. The center of the star consists of a large fact table and the points of the star are the dimension tables.A star query is a join between a fact table and a number of dimension tables. Each dimension table is joined to the fact table using a primary key to foreign key join, but the dimension tables are not joined to each other. The optimizer recognizes star queries and generates efficient execution plans for them. It is not mandatory to have any foreign keys on the fact table for star transformation to take effect. A star join is a primary key to foreign key join of the dimension tables to a fact table. The main advantages of star schemas are that they:

- Provide a direct and intuitive mapping between the business entities being analyzed by end users and the schema design.

- Provide highly optimized performance for typical star queries.

- $\quad$ Are widely supported by a large number of business intelligence tools, which may anticipate or even require that the data warehouse schema contain dimension tables.

Star schemas are used for both simple data marts and very large data warehouses.

\section{SNOWFLAKE SCHEMAS}

The snowflake schema is a more complex data warehouse model than a star schema, and is a type of star schema [6]. It is called a snowflake schema because the diagram of the schema resembles a snowflake. Snowflake schemas normalize dimensions to eliminate redundancy i. e., the dimension data has been grouped into multiple tables instead of one large table. While this saves space, it increases the number of dimension tables and requires more foreign key joins. The result is more complex queries and reduced query performance. The main advantages of Snowflake schemas are that they:

- $\quad$ save memory space for data.

- $\quad$ increases the number of dimension tables and requires more foreign key joins.

- the result is more complex queries.

\subsection{Third Normal Form (3NF)}

Third normal form modeling is a classical relational-database modeling technique that minimizes data redundancy through normalization [6]. When compared to a star schema, a $3 \mathrm{NF}$ schema typically has a larger number of tables due to this normalization process. $3 \mathrm{NF}$ schemas are typically chosen for large data warehouses, especially environments with significant data-loading requirements that are used to feed data marts and execute long-running queries. The main advantages of $3 \mathrm{NF}$ schemas are that they:
- Provide a neutral schema design, independent of any application or data-usage considerations

- May require less data-transformation than more normalized schemas such as star schemas

\section{DATA WAREHOUSE DESIGN}

An example of a record in a fact table for an Examination Automation System for a University, on a single event,such as a result of a Student at a particular session of an Academic year at Under/Post Graduate Level, has been considered.

In addition to the fact tables Table 1 , there are also dimension tables in the database. These dimension tables describe the options to "cut" or view the data in the fact table. The star and snowflake schemas all use more than one dimension table in their database [2][3]. The records in a single dimension table represent the levels or choices of aggregation for the given dimension [7][17]. The classic data warehouse example used is the Result dimension[10][12]. The records in the Result dimension table will indicate that the fact table data can be aggregated by Subjects assigned, Enrollment of Students, Marks Obtained etc. Another dimension would be date. Using the date dimension we would be able to analyze data by a single date or dates aggregated by month, quarter, fiscal year, calendar year, holidays, etc.

For an Examination Automation System, a simple fact table would have the following column variables is show in table below.

Table 1: Fact Dimension Details

fact_DIM

\begin{tabular}{|l|l|l|}
\hline ROLLNO key & $\begin{array}{l}\text { Rollno Pertaining } \\
\text { to a Session }\end{array}$ & Numeric \\
\hline REGNO key & $\begin{array}{l}\text { Registration No. of } \\
\text { Student }\end{array}$ & Alphanumeric \\
\hline RESULT & Calculated Result & Alphanumeric \\
\hline TOTALM & Total Marks & Numeric \\
\hline RESGAZ & $\begin{array}{l}\text { Showing Statue } \\
\text { Result }\end{array}$ & Alphanumeric \\
\hline Sesson_ID Key & $\begin{array}{l}\text { Session of } \\
\text { Examination FKey }\end{array}$ & Alphanumeric \\
\hline college_id Key & College Code FKey & Alphanumeric \\
\hline dateID Key & Date FKey & Alphanumeric \\
\hline course_code Key & Course Opted FKey & Alphanumeric \\
\hline facultyID Key & $\begin{array}{l}\text { Faculty Opted } \\
\text { FKey }\end{array}$ & Alphanumeric \\
\hline groupID Key & $\begin{array}{l}\text { Subject Group } \\
\text { Table FKey }\end{array}$ & Alphanumeric \\
\hline
\end{tabular}

\section{STAR JOIN SCHEMA}

The star join schema (also known as the star schema) is a database in which there is a single fact table and many dimension tables. These tables are not normalized. They are unlike traditional operational data bases where one attempts to normalize the tables [10][14]. In the fact table there is one segment for each dimension. The fact table uses a compound 
key made up of the group of the dimensions. In addition, the fact table usually contains additional variables which typically are additive numbers, i.e., numericfacts. In our Examination Automation System example the individual dimension table would capture views by:

$>$ Enrollment containing registration no, name and parentage

$>$ Subjects taken by the student

$>$ Student enrolled in the course

$>$ Marks obtained in every subject

$>$ Date of declaration, session, year

$>$ College information

$>$ Course information

$>$ Faculty details etc.

For the full star schema of Examination Automation System see Appendix 1 at the end of the paper.

\section{USING THE STAR SCHEMA FOR BUILDING DATASETS}

Users of the Examination Automation System will want tolook at the data summarized to various levels. Joining selected dimension tables to the fact table will provide the user with a dataset on which to aggregate the needed information [1].

For example, to generate the result of the student would require a the join of five tables namely Fact Table, EnrollmentDimension Table, CourseDimension Table subject_groupsDimension Table and marksDimension Table. The resultant data file will then be aggregated by using the Proc Summary step to produce a dataset for analysis. Below is a demonstration of this approach.

Table 3: Client Statistics for the above query resulted in the following details.

\begin{tabular}{|l|l|l|l|l|}
\hline Client Statistics Information & Trial 3 & Trial 2 & Trail 1 & Average \\
\hline Client Execution Time & $20: 25: 51$ & $20: 25: 46$ & $20: 25: 36$ & \\
\hline Query Profile Statistics & 27453 & 27453 & 27453 & 27453 \\
\hline Rows returned by SELECT statements & \multicolumn{5}{l|}{} \\
\hline Network Statistics & 3 & 3 & 3 & 3 \\
\hline Number of server roundtrips & 3 & 3 & 3 & 3 \\
\hline TDS packets sent from client & 3 & 1974 & 1974 & 1974 \\
\hline TDS packets received from server & 1974 & 2220 & 2220 & 2220 \\
\hline Bytes sent from client & 2220 & 8074601 & 8074601 & 8074602 \\
\hline Bytes received from server & 8074601 & 8074 & \\
\hline Time Statistics & \multicolumn{5}{|l}{} \\
\hline Client processing time & 551 & 568 & 817 & 645.3333 \\
\hline Total execution time & 859 & 861 & 1127 & 949 \\
\hline Wait time on server replies & 308 & 293 & 310 & 303.6667 \\
\hline
\end{tabular}

An Examination Automation System of 2500000 records in the fact table with 12 column variables, totaling to 30 megabytes of space. The memory taken by the dimension tables are depicted in table below.

Table 2: Dimension Table records in Megabytes

\begin{tabular}{|l|l|}
\hline DIMENTION NAME & SIZE \\
\hline Enrollment & $176.388 \mathrm{MB}$ \\
\hline Subject & $0.056 \mathrm{MB}$ \\
\hline Marks & $150.5 \mathrm{MB}$ \\
\hline Course & $0.020 \mathrm{MB}$ \\
\hline College & $0.008 \mathrm{MB}$ \\
\hline Date & $0.015 \mathrm{MB}$ \\
\hline Faculty & $0.012 \mathrm{MB}$ \\
\hline Group & $0.095 \mathrm{MB}$ \\
\hline Session & $0.010 \mathrm{MB}$ \\
\hline Total Space & $\mathbf{3 2 7 . 1 0} \mathrm{MB}$ \\
\hline
\end{tabular}

\subsection{GENERATING THE FINAL RESULT NOTIFICATION}

An algorithm was devleloped and code implemented in SQL using SQL Server Management Studio Express as Front end and Microsoft SQL 2005 at the back end for testing the described schema. The results of the simulationare presented in table below. 


\section{BUILDING THE DECISION SUPPORT DATABASE}

Similarly, other datasets could be generated for analysis. Using the building blocks of the fact table and the various dimension tables, one has thousands of ways to aggregate the data. For expedient analysis purposes, frequently needed aggregated datasets should be created in advance for the users [15][16]. Having data readily and easily available is a major tenet of data warehousing. For Examination Automation System, some aggregated datasets were:

- Generating the Final Result Notification per Subject, College, Subject Groups, Year Wise, Gender etc.

- Remuneration for Paper Checkers, Checking Assistants and other Officials.

- Students Count by Age, Gender, Pass, Fail, Reappear in subject pertaining to per college, subject, year, group of subjects.

- Interests of Various of Students in Courses, Colleges, Subjects etc and Improvements to be made in the Education System etc.

- No of Students enrolled for a particular course, subject, college, courses within a college, subject within a college.

- $\quad$ Students who have passed with and without statues.

- Percentage of result, subject wise, college wise, course wise, group wise.

As one can see, the Star Schema lends itself well forCustom analysis.

\section{OLAP AND DATA MINING}

On-line Analytical Processing (OLAP) is theanalytical capabilities provided by the data warehouse or data mart. One can view granular data or variousaggregations of data for business analyses using graphical-user-friendly tools [4][18]. Data warehouse and data marts exist to answer questions and find business opportunities. There are many ways to analyze data using procedures such as ProcdecodeMks, ProcgetResult, Procfmaster, Procrollidx, Proc Tabulate.

Finally, data mining is the name given to newer statistical techniques used to explore voluminous data stores. These techniques include decision trees and neural networks. These methods, like neural networks, can sometimes handle colinearity better than the older statistical techniques.

\section{COMPARISON WITH 3RD NORMAL FORM}

A comparative study was also performed by taking same amount of test data and the observations were tabulated in the below mentioned table. It was observed that there was a big tradeoff between the memory and the speed in the implementation of 3rd Normal form and Star Schema.
Table 4: Execution Time for Result Preparation

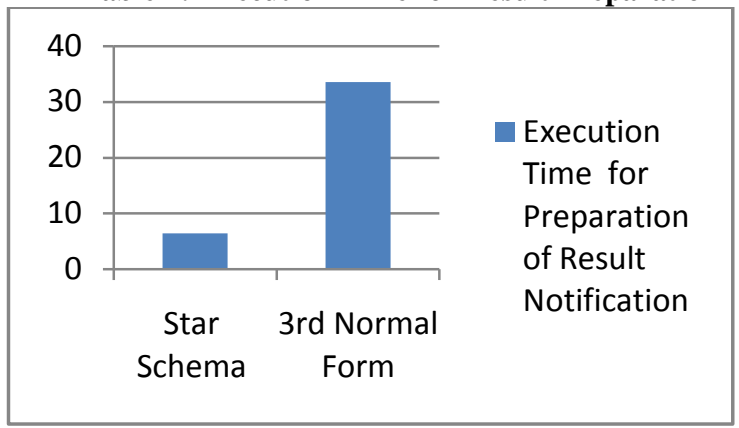

Table 5: Execution Time for Result Preparation

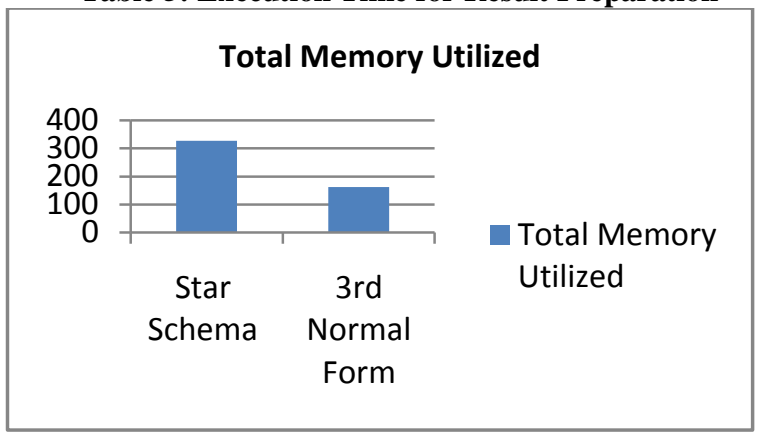

\section{CONCLUSION}

The data warehousing technology i s gaining wide attention, and many organizations are building data warehouses (or, data marts) to help them in data analysis in decision for decision support. Data Warehousing is a newly emerged field of study in Computing Sciences. Due to its viz. multidisciplinary nature, it has overlapping area of studies in three different computing disciplines. This overlapping sometimes may cause contradictory definitions for a specific concept. To overcome this problem of data warehousing for Examination Automation System,it was considered for Star Schema Design. In this regard various functionaldimensions of the Examination System were designed and connected to a Fact Transaction Dimension. Furthermore the general issues like the Client Statistics and Query Design were taken up and various Decision Support Databases weredesigned and implemented using the same star Schema.

\section{REFERENCES}

[1] A. Gupta, V. Harinarayan, and D. Quass. Aggregatequery processing in data-warehousing environments. In Proc. 21th Int. Conf. on Very Large Data Bases, Zurich, Switzerland, 1995.

[2] ACM/ IEEE-CS Joint Task Force for Computing Curriculum 2005. "Computing Curriculum 2005". The Over view report" 30 Sep, 2005

[3] C. Fahrner, and G. Vossen. A survey of database transformations based on the Entity-Relationship model. Data \& Knowledge Engineering, vol. 15, n. 3, pp. 213250. 1995.:

[4] CAI Yong, HE Guangsheng, "Designing Model of Data Warehouse with OO Method [J]", Computer Engineering and Applications, 2003.6.

[5] Inmon, W. H., "Building the Data Warehouse", Second Edition, John Wiley \& Sons, Inc 1996 
[6] Fon Silvers, "Building and Maintaining a Data Warehouse," AN AUERBACH BOOK", CRC Press is an imprint of the Taylor \& Francis Group, an informa business

[7] Jeff Lawyer, ShamsulChowdhury, “ Best Practices in Data Warehousing to Support Business Initiatives and Needs", Proceedings of the 37th Hawaii International Conference on System Sciences - 2004

[8] Jorge Bernardino, Pedro Furtado, Henrique Madeira," A Cost Effective Approach for Very Large Data Warehouses", Proceedings of the International Database Engineering and Applications Symposium, 2002

[9] Kimball, Ralph, "The Data Warehouse Toolkit: Practical Techniques for Building Dimensional Data Warehouses", John Wiley \& Sons, Inc, 1996.

[10]Krishna. "Principles of Curriculum Design and Revision: A Case Study in Implementing Computing Curricula CC2001”. ITiCSE'05, June 27-29, 2005

[11]Larry, Greenfield, LGI Systems Inc., "The Data Warehousing Information Center," 1997 pp http://pwp.starnetinc.com/larryg/index.html.

[12]LIN Yu,etc, "The Principles and Applications of Data Warehouse [M]", Posts \& Telecommunications Press, 2003.1
[13]R. Barquin, and S. Edelstein. "Planning and Designing, the Data Warehouse",. Prentice Hall, 1996.

[14]REN Jinluan, GU Peiliang, ZENG Zhenxiang, "Research on the Methods of Designing Data Structure of Data Warehouse [J]". Computer Engineering and Applications, 2001.22.

[15]SvetlozarNestorov, NenadJukic, "Ad-Hoc AssociationRule Mining within the Data Warehouse", Proceedings of the 36th Hawaii International Conference on System Sciences, 2002

[16]Syed Najam-ul-Hassan, MaqboolUddinShaikh, UzairlqbalJanjua," Data Warehousing an Academic Discipline "Curriculum Development Approach, Methodologies and Issues", 2006

[17]Wu Shuning, Cui Deguang, Cheng Peng ,"The Four-stage Standardized Modeling Method in Data

[18]Warehouse System Development" Proceedings of the IEEE International Conference on Mechatronics \& Automation Niagara Falls, Canada • July 2005

[19]YUAN Hong, HE Houcun, "Online Analysis and Data Warehouse Modeling Technologies [J]", Computer Application Research, 1999.12. 
Appendix 1

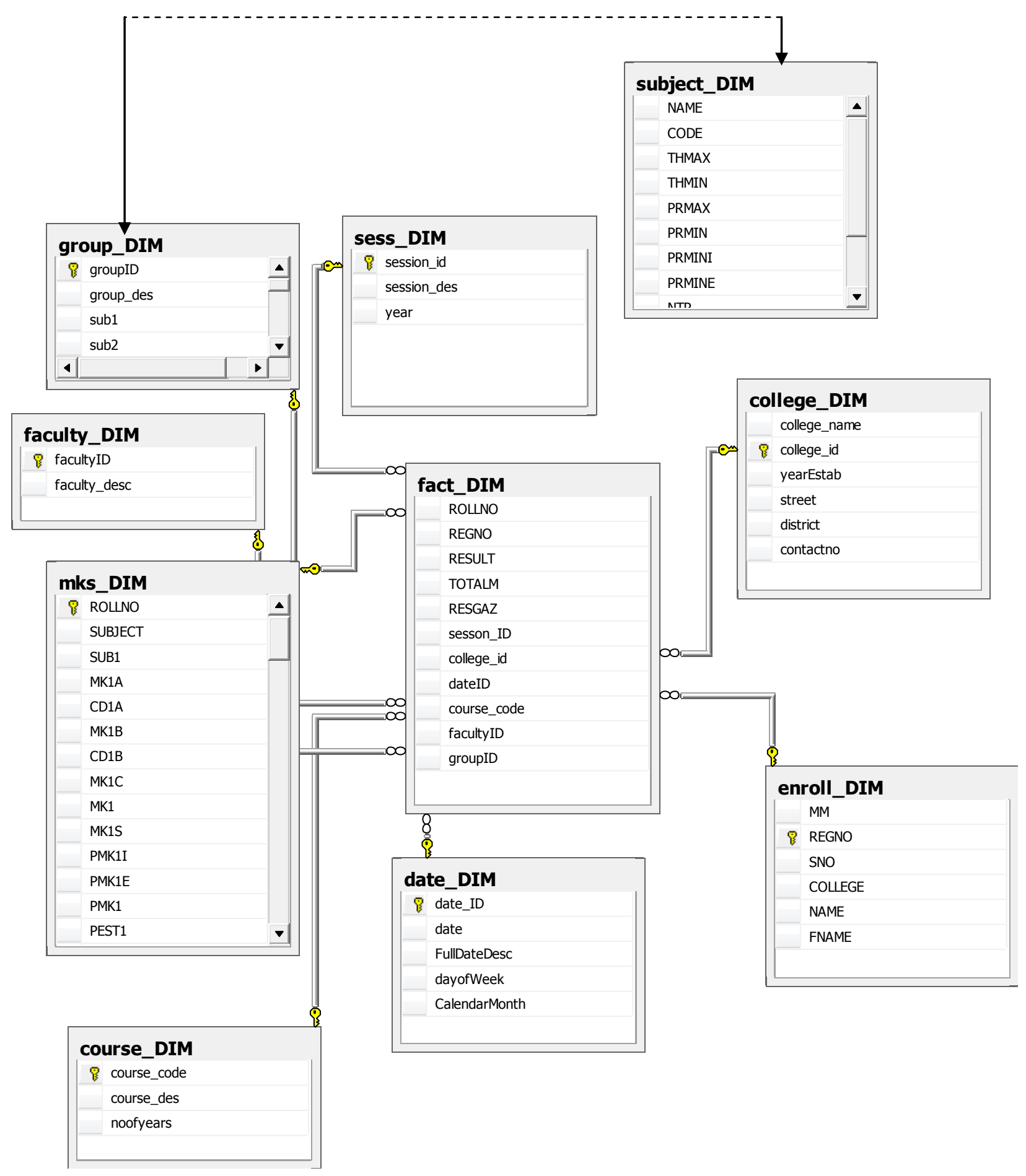

